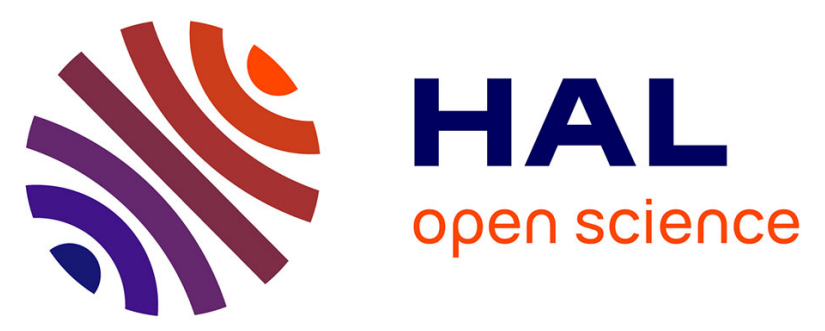

\title{
Gamma Knife radiosurgery for intravestibular and intracochlear schwannomas
}

Constantin Tuleasca, Mercy George, Luis Schiappacasse, David Patin, Jacopo Fenu, Raphael Maire, Marc Levivier

\section{- To cite this version:}

Constantin Tuleasca, Mercy George, Luis Schiappacasse, David Patin, Jacopo Fenu, et al.. Gamma Knife radiosurgery for intravestibular and intracochlear schwannomas. Acta Neurochirurgica, 2019, 161 (1), pp.63-67. 10.1007/s00701-018-3762-y · hal-02171122

\section{HAL Id: hal-02171122 \\ https://hal.sorbonne-universite.fr/hal-02171122}

Submitted on 2 Jul 2019

HAL is a multi-disciplinary open access archive for the deposit and dissemination of scientific research documents, whether they are published or not. The documents may come from teaching and research institutions in France or abroad, or from public or private research centers.
L'archive ouverte pluridisciplinaire HAL, est destinée au dépôt et à la diffusion de documents scientifiques de niveau recherche, publiés ou non, émanant des établissements d'enseignement et de recherche français ou étrangers, des laboratoires publics ou privés. 


\section{Gamma Knife radiosurgery}

\section{for intravestibular and intracochlear schwannomas}

Constantin Tuleasca, MD-PhD ${ }^{1,2,3,4,5}$, Mercy George, $\mathrm{MD}^{6}$, Luis Schiappacasse, $\mathrm{MD}^{7}$, David Patin, $\mathrm{PhD}^{8}$, Jacopo Fenu ${ }^{9}$, Raphael Maire, $\mathrm{MD}^{6}$, Marc Levivier, MD, PhD, IFAANS ${ }^{1,2}$

${ }^{1}$ Centre Hospitalier Universitaire Vaudois (CHUV), Neurosurgery Service and Gamma Knife Center, Lausanne, Switzerland;

${ }^{2}$ Université de Lausanne (Unil), Faculté de Biologie et de Médecine (FBM);

${ }^{3}$ Signal Processing Laboratory (LTS 5), Swiss Federal Institute of Technology (EPFL);

${ }^{4}$ Sorbonné Université, Faculté de Médecine, Paris, France;

${ }^{5}$ Assisstance Publique, Hôpitaux de Paris, Centre Hospitalier Universitaire Kremlin-Bicêtre, Service de Neurochirurgie, Le Kremlin-Bicêtre, France;

${ }^{6}$ Centre Hospitalier Universitaire Vaudois (CHUV), ENT Department, Lausanne, Switzerland

${ }^{7}$ Centre Hospitalier Universitaire Vaudois (CHUV), Radiation Oncology Department, Lausanne, Switzerland

${ }^{8}$ Institute of Radiation Physics, Lausanne, Switzerland

9 "Victor Babes" University of Medicine and Pharmacy

\section{Corresponding author:}

Constantin Tuleasca, MD-PhD, Centre Hospitalier Universitaire Vaudois, Neurosurgery Service and Gamma Knife Center and University of Lausanne, Faculty of Medicine, Rue du Bugnon 44-46, BH-08, CH-1011, Lausanne, Switzerland; Assisstance Publique, Hôpitaux de Paris, Centre Hospitalier Universitaire Kremlin-Bicêtre, Service de Neurochirurgie, Le Kremlin-Bicêtre and Sorbonne Université, Faculté de Médecine, Paris, France Tel: +41-21-314-26-02; Fax: +41-21-314-11-99; e-mail: constantin.tuleasca@gmail.com; 


\begin{abstract}

\section{Background}

Schwannomas of the VIII ${ }^{\text {th }}$ cranial nerve are benign tumours, with vast majority occurring in vestibular division. Rarely, can also arise from distal branches of cochlear, superior or inferior vestibular. We review our experience with Gamma Knife radiosurgery (GKR), as first intention treatment for intracochlear (ICS) and intravestibular (IVS) schwannomas.
\end{abstract}

\title{
Methods
}

A total number of 5 patients were analysed, treated over 8 years, between June 2010 and September 2018, with Leksell Gamma Knife Perfexion or Icon (Elekta Instruments, AB, Sweden). The marginal dose prescribed was 12 Gy at a mean prescription isodose line of 61.4\% (range 50-70). Clinical evaluation included auditory and facial function.

\section{Results}

The mean age was 49.9 (range 34-63). The mean follow-up period was 52.8 months (range 12-84). The mean target volume (TV) was $0.087 \mathrm{ml}$ (range 0.014-0.281). The mean maximal dose received by the cochlea was 11.2 Gy (range 2.6-20.3). The mean marginal dose received by the vestibule (e.g. utricula) was 14.2 Gy (range 3.8-17.5). No patient experienced an acute or subacute clinical adverse radiation effect after GKR. Four cases had overall symptom stability. In one patient (1/5), the vertigo, which was the main clinical complain, disappeared one year after GKR. However, it reappeared 3 years latter, with same pretherapeutic characteristics and is currently fluctuating. One patient experienced hearing decrease after GKR, during the first 12 months. This case received 11.2 Gy to the cochlea. Follow-up MRI course showed a decrease in size in four patients, and stability in one.

\section{Conclusions}

Gamma Knife radiosurgery is a valuable first intention treatment for ICS or IVS, in selected cases. Special attention should be paid for the dose delivered to the cochlea and the vestibular apparatus. Acute and subacute clinical effects are exceptional, while tumour control was achieved in all cases in our small series.

Key words: schwannoma, intracochlear, intralabyrinthine, radiosurgery, Gamma Knife 


\section{INTRODUCTION}

Schwannomas of the VIII ${ }^{\text {th }}$ cranial nerve are benign tumours, occurring usually in the cistern of the cerebello-pontine angle or in the internal auditory canal (IAC)[22]. They develop from the Schwann cells that from the myelin sheets of the nerves. They may originate anywhere within the course of the cochlea-vestibular nerves, peripheral to the glial-Schwann junction[7]. The vast majority arise in the vestibular division. Rarely, they can also arise from very distal branches of the cochlear, superior or inferior vestibular, at the level of the sensory end organs[18].

Intracochlear (ICS) and intravestibular (IVS) schwannomas may present with nonspecific symptoms. Usually, patients might experience complete loss, which might occur acutely or progressively, balance problems (dizziness, vertigo, postural instability) or tinnitus[16]. Clinical diagnosis is usually made by ENT-specific examination, including ear microscopy, audiological assessment, neuro-otological diagnosis with testing of the semicircular canal and the otolith organs[16]. The former is classically completed by the gold standard high-resolution magnetic resonance imaging (MRI)[1].

The common management is "wait-and-scan" strategy or, in reserved cases, different surgical approaches[2, 6, 12]. There is only one study, up-to-date, reporting the use of Gamma Knife radiosurgery (GKR) for IVS[13].

The purpose of the present study was to evaluate the safety and efficacy of GKR as a first intention treatment in selected cases of ICS and IVS.

\section{MATERIALS AND METHODS}

\section{Type of study}

The study was designed as opened, retrospective, non-randomized. A case-report form was created since the first treated patient and prospectively filled in with the baseline and follow-up data (historical cohort analysis).

\section{Participants}

Vestibular schwannoma (VS) is one of the most frequent pathology treated in our Gamma Knife Center, accounting for almost $30 \%$ of the patients. All cases with an ICS or IVS were included in the study. A total number of 5 patients were further analysed, treated over of period of 8 years, between June 2010 and September 2018. The main indication for GKR was clinical and/o radiological evolutivity. 


\section{Baseline and follow-up monitoring}

The baseline assessment included clinical demographic data, full clinical examination including of the CN. Paraclinical exam included vocal and tonal audiometry, caloric testing as well as brain MRI. After GKS, patients were evaluated at 6, 12, 24, 36, 60 and 72 months, respectively, with ENT outpatient's tests, brain MRI and neurosurgery consultation, in our office.

The auditory function was analysed by using the Gardner Robertson (GR) classification[3]. Facial function was assessed using the House-Brackmann scale[11].

\section{Description of the GKS technique}

We applied, in every case, the Leksell Model G stereotactic frame (Elekta Instruments $\mathrm{AB}$, Sweden) under local anaesthesia. Afterwards, all patients underwent stereotactic MRI and bone $\mathrm{CT}$ for target and organs at risk definition.

During dosimetry planning, special attention was given to the dose received by the cochlea in patients with functional hearing at baseline[14]. Due to the particular anatomical location, we also defined the vestibule, as the structure that is very close anatomically to the cochlea, and which might be subjected to dose increase when limiting the dose to the cochlea, as in our previously described methodology[21].

The stereotactic MRI sequences used were T1 with and without Gadolinium enhancement and T2 CISS/Fiesta without contrast, the former for a better visualization of the cranial nerves and of the cochlear and vestibular structures[8, 9]. Bone CT routinely supplemented the neuroimaging investigation in order to correct any distortion errors that might be encountered on the MRI images. Additionally, it provided important information with regard to the bony landmarks of the internal auditory canal, as well as of the cochlea and the vestibule, important in the context of our study. Particular attention was paid to the dose received by the cochlea, due to its impact for further hearing preservation[14, 19].

Leksell Gamma Knife Perfexion had been used between June 2010 and June 2016 and the Leksell Gamma Knife ICON afterwards (Elekta Instruments, AB, Sweden).

\section{Basic demographic data (Table 1)}

The mean age in this series was 49.9 (range 34-63). The mean follow-up period was 52.8 months (range 12-84). The male to female ratio was 2 to 3 . The most common symptom 
at discovery was hearing loss (4/5 patients), with one patient experiencing brutal hearing loss. One patient had vertigo (1/5) at initial clinical evaluation.

The corresponding GR class was 2 in two cases, 3 in one case, and 5 in two cases. No patient has facial palsy. The MRI appearance corresponded to a pure IVS in two cases, pure ILC in one case, and mixed IVS and ICS or with an additional cisternal part, each with one case.

\section{Basic Dosimetric data (Table 2)}

The marginal dose prescribed was 12 Gy in all cases, at a mean prescription isodose line of $61.4 \%$ (range 50-70). The mean target volume (TV) was $0.087 \mathrm{ml}$ (range 0.014-0.281). The mean prescription isodose volume (PIV) was $0.174 \mathrm{ml}$ (range 0.053-0.504).

The mean maximal dose received by the cochlea was 11.2 Gy (range 2.6-20.3). The mean marginal dose received by the vestibule (e.g. utricula) was 14.2 Gy (range 3.8-17.5).

\section{RESULTS}

\section{Clinical follow-up}

No patient experienced an acute or subacute clinical adverse radiation effect after GKR.

Four cases had overall symptom stability. In one patient (1/4), the vertigo, which was the main clinical complain, disappeared one year after GKR. However, it reappeared 3 years latter, with the same pretherapeutic characteristics and is currently fluctuating. One patient experienced hearing decrease after GKR, during the first 12 months. This case received 11.2 Gy to the cochlea.

\section{Radiological follow-up}

Follow-up MRI course showed a decrease in size in four patients, and stability in one.

\section{DISCUSSION}

To the best of our knowledge, this is the second study analysing the role of GKR as a first intention treatment for selected cases of ICS and IVS, after a previous report published earlier in 2011[13]. Beside the previous, only a small number of papers have described this particular and rare pathology, in non-radiosurgical setting, in the frame of case reports or 
small series[10]. In our experience, we report no acute or subacute adverse radiation effects in this indication, both for ICS and for IVS, as we have previously reported in our overall series of VSs treated with GKR[21]. Symptom stability was confirmed on four out of five cases. One patient, who received 11.2 Gy to the cochlea, due to the particular anatomical location and the dosimetric conditions, reported hearing decrease during follow-up course. Tumour decrease was attained in 4 cases, and stability in one.

The most commonly used classification for ICS and IVS is the one proposed by Salzmann et al.[17], separating 6 types: intracochlear (limited to the cochlea), transmodiolar (extension to IAC via modiolus), intravestibular (vestibule, with or without extension to semicircular canals), transmacular (vestibule, with or without extension to semi-circular canals and extension to IAC macula cribrosa), intravestibulocochlear (vestibule, with or without extension to semicircular canals and in the cochlea), transotic $+/$ - the cerebello-pontine angle (tumor in the cochlea, and/or vestibular portion of the inner ear, extension into the middle ear). It is well known that schwann cells of the cochlear axons in the cochlear nerve are present in the modiolus, proximal to the spiral ganglion[23, 24]. In this context, a schwannoma could develop in the modiulus, present further growth, occupy the cochlear basal turn, erode the cribriform area of the IAM and spread into the vestibule.

The classical symptom is hearing loss, followed by vertigo and imbalance[1].

During the past decades, the MRI development has allowed a fine diagnosis in this particular and rare pathology. Dedicated sequences, including T2 CISS/Fiesta images, have provided detailed anatomical description and enabled early diagnosis. This was previously most probably responsible for an underestimation of the real number of cases. The most important differential diagnosis is an infectious and inflammatory process, as ICS especially could also miming this, due to the filling defect present on T2-weighted images[1].

Once diagnosed, the management of the ICL and IVS includes: wait-and-scan strategy (patients without specific complains including good level of hearing, absence of vertigo/balance issues and tumour stability)[4], surgery, or SRS and particularly GKR, due to its steep gradient, particularly necessary in this indication, due to the presence of organs at risk. The management strategy depends on degree of symptoms and further hearing loss, tumour's size, tumour's increase, special concerns about the pathophysiological diagnosis (if surgery is performed) and particular comorbidities[5, 23, 24]. One has to keep in mind that surgical removal is followed by complete hearing loss, due to the transotic, translabyrinthine or trancanal labyrintectomy accesses[15]. 
Radiosurgery and particularly GKR have the advantage of its minimal invasiveness. With high rates of tumour control[20] and keeping the maximal dose delivered to the cochlea below 4 Gy if the anatomical conditions will allow, one might also expect hearing preservation on long-term basis[14, 19]. No vestibular deterioration has been seen in this small series.

Stereotactic radiosurgery is a valuable option as first line treatment for ICS and IVS. Technically, the procedure is feasible, while prescribing low radiation doses, such as in the more classical VSs in general (e.g. 12 Gy). Grace to the steep gradient of GKR, one could also expect functional preservation in cases having pretherapeutically high level of hearing. Tumour control was achieved in all cases in our small series.

Figure 1: Illustration of five individual cases treated in our center; the dosimetry is coloured in yellow; for displaying both the bony landmarks and the brain/tumour extension, are used fused images using the "Fuse" module inside the Leksell Gamma Plan (LGP, Elekta Instruments, $\mathrm{AB}$, Sweden); patients are quoted from $\mathrm{A}$ to $\mathrm{E}$

Figure 2: Illustrative case of an IVS, with the images at the time of Gamma Knife (A), and 3 years later (B, with superimposed dosimetry), showing the shrinkage of the tumour

Table 1: Basic demographic data

Table 2: Basic dosimetric data

\section{Compliance with Ethical Standards:}

Funding: This study was funded by Lausanne University Hospital.

Conflict of Interest: The authors report no conflict of interest.

Ethical approval: All procedures performed in studies involving human participants were in accordance with the ethical standards of the institutional and/or national research committee and with the 1964 Helsinki declaration and its later amendments or comparable ethical standards.

Ethical approval: For this type of study formal consent is not required 


\section{References}

1. Bittencourt AG, Alves RD, Ikari LS, Burke PR, Gebrim EM, Bento RF (2014) Intracochlear schwannoma: diagnosis and management. International archives of otorhinolaryngology 18:322-324

2. Di Lella F, Dispenza F, De Stefano A, Falcioni M, Sanna M (2007) Management of intralabyrinthine schwannomas. Auris, nasus, larynx 34:459-463

3. Gardner G, Robertson JH (1988) Hearing preservation in unilateral acoustic neuroma surgery. The Annals of otology, rhinology, and laryngology 97:55-66 4. Gosselin E, Maniakas A, Saliba I (2016) Meta-analysis on the clinical outcomes in patients with intralabyrinthine schwannomas: conservative management vs. microsurgery. European archives of oto-rhino-laryngology : official journal of the European Federation of Oto-Rhino-Laryngological Societies 273:1357-1367 5. Grayeli AB, Fond C, Kalamarides M, Bouccara D, Cazals-Hatem D, Cyna-Gorse F, Sterkers $O$ (2007) Diagnosis and management of intracochlear schwannomas. Otology \& neurotology : official publication of the American Otological Society, American Neurotology Society [and] European Academy of Otology and Neurotology 28:951-957 6. Green JD, Jr., McKenzie JD (1999) Diagnosis and management of intralabyrinthine schwannomas. The Laryngoscope 109:1626-1631

7. Hamed A, Linthicum FH, Jr. (2005) Intralabyrinthine schwannoma. Otology \& neurotology : official publication of the American Otological Society, American Neurotology Society [and] European Academy of Otology and Neurotology 26:10851086

8. Hayashi M, Chernov M, Tamura N, Izawa M, Muragaki Y, Iseki H, Okada Y, Takakura K (2011) Gamma knife robotic microradiosurgery for benign skull base meningiomas: tumor shrinkage may depend on the amount of radiation energy delivered per lesion volume (unit energy). Stereotact Funct Neurosurg 89:6-16 9. Hayashi M, Ochiai T, Nakaya K, Chernov M, Tamura N, Yomo S, Izawa M, Hori T, Takakura K, Regis J (2006) Image-guided microradiosurgery for skull base tumors: advantages of using gadolinium-enhanced constructive interference in steady-state imaging. J Neurosurg 105 Suppl:12-17

10. Hoshino T, Ishii D (1972) Intralabyrinthine neurilemmoma. A histopathological report. ORL; journal for oto-rhino-laryngology and its related specialties 34:117-123

11. House JW, Brackmann DE (1985) Facial nerve grading system. Otolaryngology-head and neck surgery : official journal of American Academy of Otolaryngology-Head and Neck Surgery 93:146-147

12. Kennedy RJ, Shelton C, Salzman KL, Davidson HC, Harnsberger HR (2004) Intralabyrinthine schwannomas: diagnosis, management, and a new classification system. Otology \& neurotology : official publication of the American Otological Society, American Neurotology Society [and] European Academy of Otology and Neurotology 25:160-167

13. Massager N, Drogba L, Delbrouck C, Benmebarek N, Desmedt F, Devriendt D (2011) Gamma knife radiosurgery for intralabyrinthine schwannomas. Journal of radiosurgery and SBRT 1:237-245

14. Massager N, Nissim O, Delbrouck C, Delpierre I, Devriendt D, Desmedt F, Wikler D, Brotchi J, Levivier M (2007) Irradiation of cochlear structures during vestibular schwannoma radiosurgery and associated hearing outcome. Journal of neurosurgery 107:733-739 
15. Miller ME, Moriarty JM, Linetsky M, Lai C, Ishiyama A (2012) Intracochlear schwannoma presenting as diffuse cochlear enhancement: diagnostic challenges of a rare cause of deafness. Irish journal of medical science 181:131-134

16. Plontke SK, Rahne T, Pfister M, Gotze G, Heider C, Pazaitis N, Strauss C, CayeThomasen P, Kosling S (2017) Intralabyrinthine schwannomas : Surgical management and hearing rehabilitation with cochlear implants. Hno 65:136-148

17. Salzman KL, Childs AM, Davidson HC, Kennedy RJ, Shelton C, Harnsberger HR (2012) Intralabyrinthine schwannomas: imaging diagnosis and classification. AJNR. American journal of neuroradiology 33:104-109

18. Shin YR, Choi SJ, Park K, Choung YH (2009) Intralabyrinthine schwannoma involving the cochlea, vestibule, and internal auditory canal: 'canalolabyrinthine schwannoma'. European archives of oto-rhino-laryngology : official journal of the European Federation of Oto-Rhino-Laryngological Societies 266:143-145

19. Tamura M, Carron R, Yomo S, Arkha Y, Muraciolle X, Porcheron D, Thomassin JM, Roche PH, Regis J (2009) Hearing preservation after gamma knife radiosurgery for vestibular schwannomas presenting with high-level hearing. Neurosurgery 64:289-296; discussion 296

20. Tsao MN, Sahgal A, Xu W, De Salles A, Hayashi M, Levivier M, Ma L, Martinez R, Regis J, Ryu S, Slotman BJ, Paddick I (2017) Stereotactic radiosurgery for vestibular schwannoma: International Stereotactic Radiosurgery Society (ISRS) Practice Guideline. Journal of radiosurgery and SBRT 5:5-24

21. Tuleasca C, George M, Faouzi M, Schiappacasse L, Leroy HA, Zeverino M, Daniel RT, Maire R, Levivier M (2016) Acute clinical adverse radiation effects after Gamma Knife surgery for vestibular schwannomas. Journal of neurosurgery 125:73-82

22. Tuleasca C, Levivier M (2018) A virtual musician quartet with vestibular schwannoma. The Lancet. Oncology 19:1025

23. Yoshida T, Sone M, Naganawa S, Nakashima T (2011) Accuracy of 3.0 Tesla magnetic resonance imaging in the diagnosis of intracochlear schwannoma. Auris, nasus, larynx 38:551-554

24. Zhu AF, McKinnon BJ (2012) Transcanal surgical excision of an intracochlear schwannoma. American journal of otolaryngology 33:779-781 

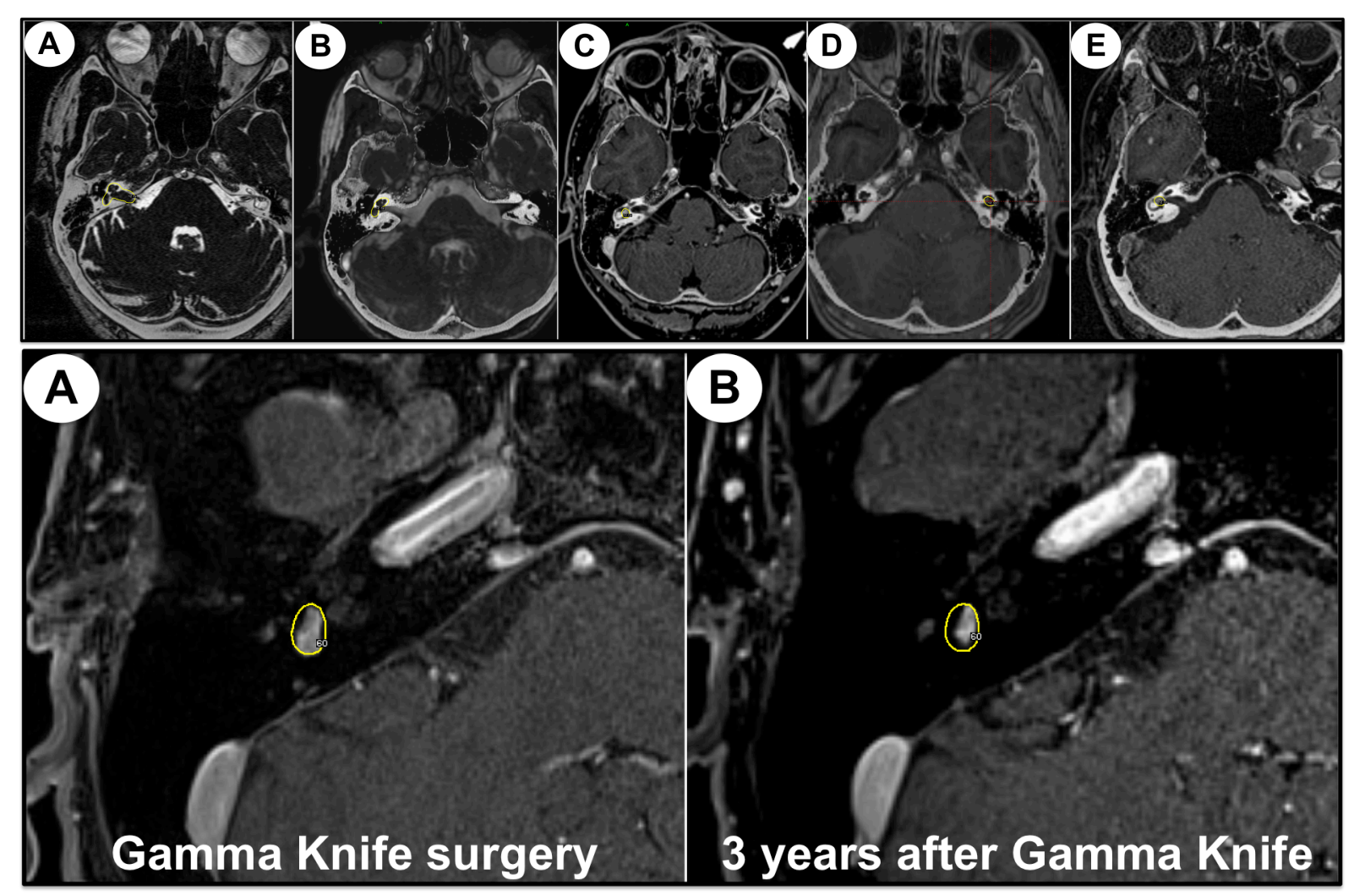